\title{
RETICULOCYTES AS A MARKER OF OXYGEN TRANSPORT SYSTEM ADAPTATION TO PHYSICAL ACTIVITY IN ENDURANCE SPORTS
}

\author{
Iryna Rybina \\ Republican Scientific and Practical Center Of Sports, Minsk, Belarus
}

\begin{abstract}
Backgraund. Peripheral blood reticulocytes and aging of the activity of these cells are markers of erythropoiesis activity. The purpose of this study was to examine changes of reticulocytes in blood formation of elite athletes in endurance sports during the preparation and the competitive periods.

Methods. Reticulocytes and IRF content was examined in blood of 305 elite athletes aged 20-26 years that specialized in swimming, biathlon, cross-country skiing, kayaking and canoeing, rowing and cycling. At various stages of preparation 459 blood tests were performed and processed. Capillary blood was analysed using a haematology analyser SYSMEX XT 2000i.

Results. Differences in reticulocytes depending on sport, gender and qualification were not found. Increase in reticulocytes was marked for male athletes in the age aspect, for females, reduction was observed. A tendency of average reticulocytes group data in peripheral blood at various stages of the annual preparation cycle was found: reduction of the amount of reticulocytes in competition period. This pattern was found regardless of gender. Similar evidence was found concerning the dynamics of IRF at the stages of the annual training.

Conclusion. The effect of training activity on reticulocyte indices in the annual training cycle is related to the direction, duration and intensity of physical activity in different periods of training. Changes in reticulocytic indices may be due to effects such as hemoconcentration and hemodilution appearing after exercise in the corresponding areas of energy supply.
\end{abstract}

Keywords: reticulocytes, erythropoiesis, elite athletes, training loads.

\section{INTRODUCTION}

$\Lambda$ leading role in energy supply of physical activity in endurance sports belongs to aerobic processes, where the intensity of muscular activity depends significantly on oxygen circulatory function of blood. Oxygen circulation depends on erythropoiesis activity, the rate of which can identify early tiredness symptoms.

Peripheral blood reticulocytes and aging of the activity of these cells are markers of erythropoiesis activity. The amount of blood reticulocytes and their sub-populations of different age reflect the regenerative ability of bone marrow and is an important marker for monitoring training (Asshenden, Lacoste, Orhant, Audran, \& Sharpe, 2004; Banfi, 2008; Fallon \& Bishop, 2002;
Lombardi, Colombini, Lanteri, \& Banfi, 2013; Nadarajan, Ooi, Sthaneshwar, \& Thompson, 2009).

Reticulocytes content in ribonucleic acids remain, mitochondria and other cell organelles are destroyed as they age, and the cells are transformed into mature red blood cells. Oxygen transfer efficiency of younger cells is slightly lower compared to mature erythrocytes.

Athletes' biological passport model includes parameters of peripheral blood reticulocytes, which promoted interest in studying the influence of training and competition on reticulocytes. Relatively little information is published in specialized periodicals concerning behaviour of reticulocytes depending on training and 
competitive physical activity. Some studies suggest that a change in reticulocytes depends on sport specifics as well as seasonal factors related to training process and schedule of events. Certain studies mark fluctuations in reticulocytes during the season from 5 to 21\% (Lombardi at al., 2013; Banfi, Lundby, Robach, \& Lippi, 2011).

The results of reticulocytes studies in different periods of training show a tendency to decrease the amount of reticulocytes from preparatory to competitive period (Banfi, 2008). The amount of reticulocytes in different sports can vary, but in most cases there is either a decrease of this parameter before a competition period, or the absence of any dynamics (Banfi \& Del Fabbro, 2007). Immature reticulocyte fraction (IRF) does not undergo significant fluctuations in different periods of the training cycle. There is evidence of an increase of IRF for cyclic sport athletes during the competitive season. In general, available literature describes multidirectional dynamics of reticulocytes under the influence of intense muscular activity. A study of changes in reticulocytes under the influence of the training process has both scientific and practical interest.

The purpose of this study was to examine changes in reticulocytes in blood formation of elite athletes in endurance sports during the preparation and the competitive periods.

\section{METHODS}

Reticulocytes and IRF content were examined in blood of 305 elite athletes aged 19-26 years that specialized in swimming, biathlon, cross-country skiing, kayaking and canoeing, rowing and cycling. At various stages of preparation 459 blood tests were performed and processed. Capillary blood testing was carried out using a haematology analyser SYSMEX XT 2000i. The data were tested for Gaussian distribution using the KolmogorovSmirnov test. The Mann-Whitney test for nonparametric distribution was used to determine the differences between groups.

\section{RESULTS}

Table 1 shows the ranges of reticulocytes and their fractions of immature forms in endurance sports. As the data show, no statistically significant differences in the studied parameters depending on sport as well as gender and qualification were revealed.
Table 1. Reticulocytes (\%) and IRF in peripheral blood of elite athletes in cyclic sports $(X \pm \sigma)$

\begin{tabular}{|l|c|c|c|c|c|c|}
\hline \multirow{2}{*}{\multicolumn{1}{|c}{ Cyclic sport }} & \multicolumn{3}{c|}{ Men } & \multicolumn{3}{c|}{ Women } \\
\cline { 2 - 7 } & $n$ & Reticulocytes (\%) & IRF & $n$ & Reticulocytes (\%) & IRF \\
\hline Biathlon & 12 & $0.60 \pm 0.20$ & $3.28 \pm 1.74$ & 19 & $0.64 \pm 0.31$ & $2.10 \pm 1.68$ \\
\hline Cross-country skiing & 48 & $0.51 \pm 0.23$ & $2.83 \pm 1.59$ & 18 & $0.58 \pm 0.16$ & $1.77 \pm 1.36$ \\
\hline Swimming & 56 & $0.64 \pm 0.21$ & $3.98 \pm 2.32$ & 92 & $0.61 \pm 0.23$ & $3.06 \pm 2.07$ \\
\hline Rowing & 63 & $0.62 \pm 0.23$ & $2.54 \pm 2.02$ & 28 & $0.60 \pm 0.20$ & $3.42 \pm 2.07$ \\
\hline Kayaking and canoeing & 43 & $0.61 \pm 0.20$ & $6.21 \pm 2.78$ & 32 & $0.58 \pm 0.21$ & $3.76 \pm 2.59$ \\
\hline Cycling & 41 & $0.61 \pm 0.22$ & $2.61 \pm 1.78$ & 7 & $0.64 \pm 0.21$ & $3.53 \pm 2.08$ \\
\hline
\end{tabular}

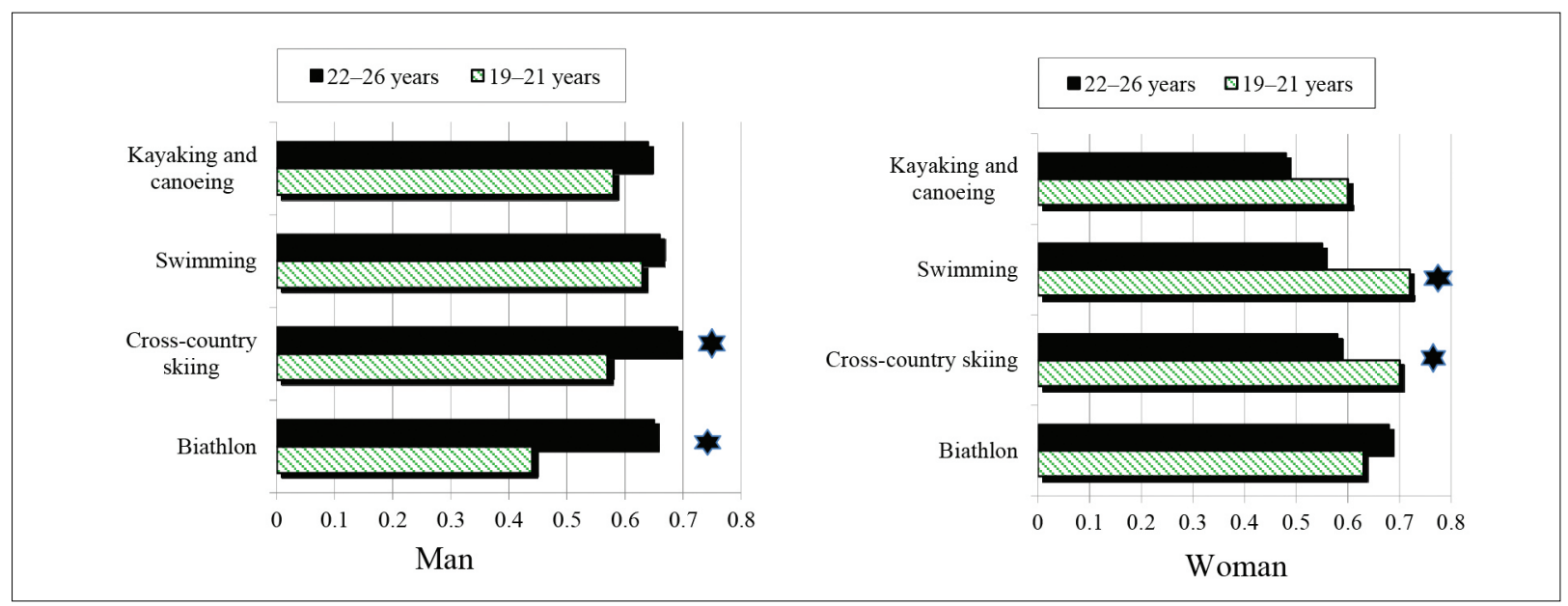

Note. $*-p<.05$.

Figure 1. The mean group data of reticulocytes in cyclic sports athletes in the aspect of age and gender 


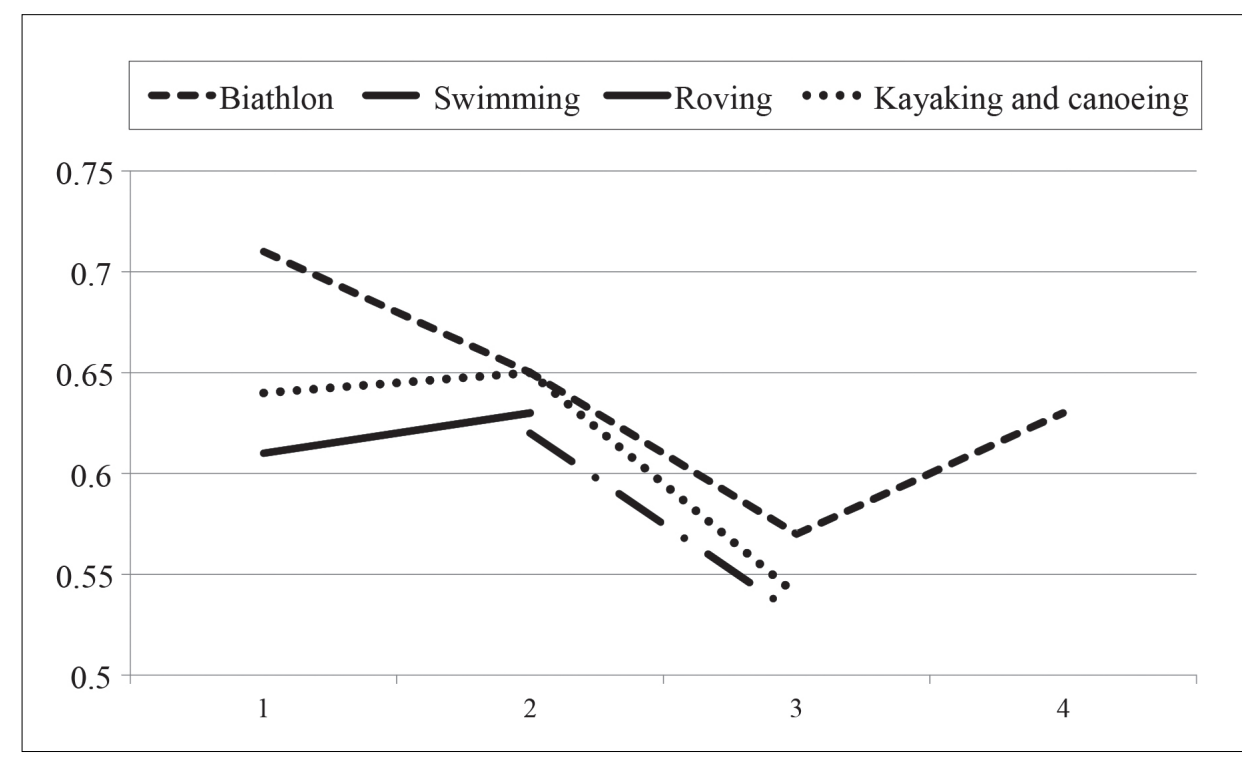

Figure 2. The dynamics of reticulocytes amount at stages in cyclic sports annual preparation cycle for males

Note. 1 - generalized stage of preparatory period, 2 - specialized stage of preparatory period, 3 - competitive, 4 - recovery.

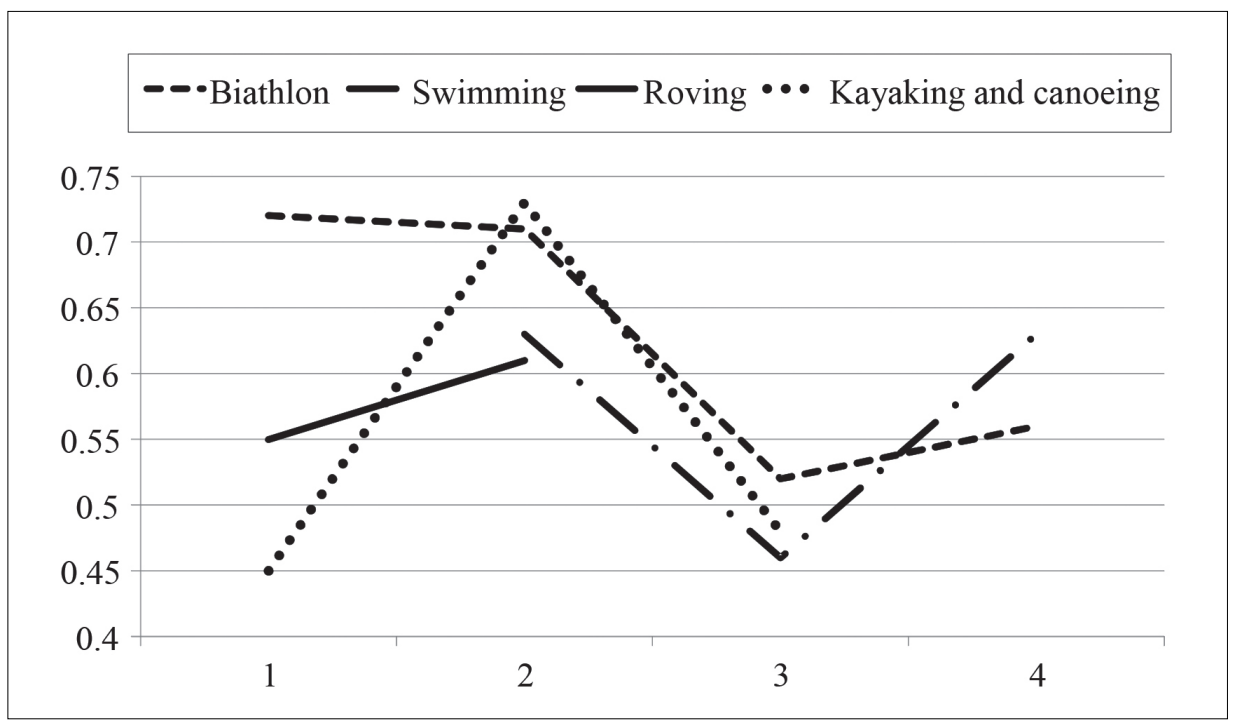

Figure 3. The dynamics of changes in reticulocytes amount at stages in cyclic sports annual preparation cycle for females

Note. 1 - generalized stage of preparatory period, 2 - specialized stage of preparatory period, 3 - competitive, 4 - recovery.

Figure 1 shows multidirectional trend of reticulocytes amount regarding the aspect of age for male and female athletes.

Figures $2-3$ present the trend line of average reticulocytes group data in peripheral blood at various stages of the annual preparation cycle.

\section{DISCUSSION}

The amount of reticulocytes may be affected by the number of factors including individual metabolism features, circadian rhythms, seasonality, physiological activity of athletes' organisms, athletes' gender and age and many others depending on muscle activity in each sport. Studies in reticulocytes amount are an important marker for detecting early signs of oxygen decrease in blood and timely pharmacological and nondrug correction of training in order to improve its efficiency (Fallon \& Bishop, 2002).
Increase in reticulocytes is marked for male athletes in the age aspect; for females, reduction is observed. This phenomenon seems to be due to differences in the dynamics of activation and inhibition of erythropoiesis in different gender groups with increasing age. Leading factor of erythropoiesis stimulation is the synthesis of erythropoietin hormone that occurs as a result of hypoxic condition.

In addition, sex hormones have a significant effect on erythropoiesis. Female sex hormones oestrogens cause inhibition of erythropoiesis. Products of metabolism of male sex hormones androgens have positive impact on erythropoiesis activation, which contributes to sensitivity increase of bone marrow to erythropoietin. The activity of erythropoiesis changes with the increasing production of sex hormones in the age aspect due to the multidirectional impact on erythropoietic function of oestrogens and androgens. 
The trend of reticulocytes amount change during the season in different sports differs, but what causes obvious interest is the common to all sports pattern of reducing the amount of reticulocytes in competition period, which is characterized by the high level of exertion of all athletes' body functioning systems including oxygen transport. This pattern is seen regardless of gender. The dynamics of IRF at the stages of the annual training is similar.

Reduction of reticulocytes amount and IRF in competitive period may be due to accelerated ageing of red blood cells and slowing of erythropoietic activity. Acceleration of red blood cell ageing may be the result of adaptation aimed at the improvement of oxygen transport by aged erythroid cells.

On the other hand, the reduction in hemopoietic system's capacity at the start of competitive period may be a consequence of large training loads during generalized and specialized stages of preparatory period. As a result of intensive muscular exertion proliferative activity of bone marrow decreases under the influence of increasing needs of erythrocyte cell production. The cause of this phenomenon may be insufficient amount of iron and serum protein for the needs of erythropoiesis, which results in its inhibition. On the one hand, iron deficiency leads to a decrease of synthesis of iron-containing proteins, and on the other hand, the lack of proteins, such as transferrin, reduces the possibility of transporting iron for erythropoietic purposes. In addition, activation of lipid peroxidation during high-intensity physical activity may contribute to damaging cell membranes of erythrocytic class.

The tendency of reticulocytes and IRF increasing from generalized to specializedpreparatory period, apparently, is the result of performing a significant amount of training loads, aimed at endurance improvement. More intensive and longer training aimed at endurance enhancement during generalized stage of preparatory period obviously leads to prolonged stimulation of bone marrow due to the process of erythrocyte haemolysis caused by training.

\section{CONCLUSION}

Studying the reticulocytic indices dynamics from peripheral blood of cyclic sports athletes allows us to estimate the impact of training and competitive processes on erythropoiesis activity intended to compensate the hypoxic state induced by the exercise.

The effect of training activity on reticulocyte indices in the annual training cycle is related to the direction, duration and intensity of physical activity in different periods of training. Changes in the reticulocytic indices may be due to effects such as hemoconcentration and hemodilution appearing after exercise in the corresponding areas of energy supply.

\section{REFERENCES}

Asshenden, M. J., Lacoste, A., Orhant, E., Audran, M., \& Sharpe, K. (2004). Longitudinal variation of hemoglobin and reticulocytes in elite rowers. Haematologica, 89(11), 1403-1404.

Banfi, G., \& Del Fabbro, M. (2007). Behaviour of reticulocyte counts and immature reticulocyte fraction during a competitive season in elite athletes of four different sports. International Journal of Laboratory Haematology, 29, 127-131. doi: 10.1111/j.1751553X.2006.00847.x

Banfi, G., Lundby, C., Robach, P., \& Lippi, G. (2011). Seasonal variations of hematological parameters in athletes. European Journal of Applied Physiology, 111(1), 9-16. doi: 10.1007/s00421-010-1641-1

Banfi, G. (2008). Reticulocytes in sports medicine. Sports Medicine, 38(3), 187-211. PMID: 18278982
Fallon, K. E., \& Bishop, G. (2002). Changes in erythropoiesis assessed by reticulocyte parameters during ultralong distance running. Clinical Journal of Sports Medicine, 12(3), 172-178. doi: 10.1097/00042752200205000-00005

Lombardi, G., Colombini, A., Lanteri, P., \& Banfi, G. (2013). Reticulocytes in sports medicine: An update. Advances in Clinical Chemistry, 59, 125-153. PMID: 23461135

Nadarajan, V. S., Ooi, C. H., Sthaneshwar, P., \& Thompson, M. W. (2009). The utility of immature reticulocyte fraction as an indicator of erythropoietic response to altitude training in elite cyclists. International Journal of Laboratory Haematology, 6, 10-12. doi: 10.1111/j.1751-553X.2008.01132.x 